\title{
Laboratory millimeter wave spectrum and astronomical search for vinyl acetate ${ }^{\star}$
}

\author{
L. Kolesniková ${ }^{1}$, I. Peña ${ }^{1}$, J. L. Alonso ${ }^{1}$, J. Cernicharo ${ }^{2}$, B. Tercero ${ }^{2}$, and I. Kleiner ${ }^{3}$ \\ ${ }^{1}$ Grupo de Espectroscopía Molecular (GEM), Edificio Quifima, Área de Química-Física, Laboratorios de Espectroscopia y \\ Bioespectroscopia, Parque Científico UVa, Unidad Asociada CSIC, Universidad de Valladolid, 47011 Valladolid, Spain \\ e-mail: lucie.kolesnikova@uva.es \\ 2 Instituto de Ciencia de Materiales de Madrid, CSIC, C/ Sor Juana Inés de la Cruz 3, 28049 Cantoblanco, Spain \\ ${ }^{3}$ Laboratoire Interuniversitaire des Systèmes Atmosphériques (LISA), CNRS/IPSL, UMR 7583, Universités Paris Est et Diderot, \\ Paris, 61 av Général de Gaulle, 94010 Créteil, France
}

Received 2 February 2015 / Accepted 16 March 2015

\section{ABSTRACT}

\begin{abstract}
Context. The recent discovery of methyl acetate in Orion $\mathrm{KL}$ makes vinyl acetate, $\mathrm{CH}_{3} \mathrm{C}=\mathrm{OOCH}=\mathrm{CH}_{2}$, a potential molecule in the interstellar medium. We obtained very accurate spectroscopic constants in a comprehensive laboratory analysis of its rotational spectra which can be used to predict those transition frequencies towards interstellar sources.

Aims. We present the experimental study and theoretical analysis of the ground torsional state of vinyl acetate in a large spectral range for astrophysical use.

Methods. The room-temperature rotational spectrum of vinyl acetate has been measured from 125 to $305 \mathrm{GHz}$ to provide direct frequencies to the astronomical community. Additional measurements have also been made using a broadband CP-FTMW spectrometer in the region of 6-18 GHz. Transition lines, corresponding to the most stable conformer, have been observed and assigned. All the rotational transitions revealed the $A-E$ splitting due to the methyl internal rotation and had to be treated with a specific internal rotation code (BELGI-Cs).

Results. We analyzed 2508 transitions up to $J^{\prime \prime}=75$ for $v_{\mathrm{t}}=0$ for the most stable conformer of vinyl acetate. The new lines were globally fitted with previously published data and 24 parameters of the Hamiltonian were accurately determined. The spectral features of vinyl acetate were then searched for in Orion KL. Using the whole line survey of Orion KL ( $80-280 \mathrm{GHz}$ ) obtained with the IRAM 30 m radio telescope we can provide only an upper limit to the column density of vinyl acetate. However, using the ALMA science verification data we obtain a tentative detection of this species that will require further search at other frequencies to confirm its presence in this high mass star forming region.
\end{abstract}

Key words. astrochemistry - ISM: molecules - submillimeter: ISM - astronomical databases: miscellaneous - line: identification ISM: individual objects: Orion KL

\section{Introduction}

The recent detection of increasingly complex molecules such as the first detection of ethyl formate $\left(\mathrm{CH}_{3} \mathrm{CH}_{2} \mathrm{OCOH}\right)$ toward Sgr B2(N) (Belloche et al. 2009, 2013) as well as the discovery of methyl acetate $\left(\mathrm{CH}_{3} \mathrm{COOCH}_{3}\right)$ and the gauche conformer of ethyl formate $\left(g-\mathrm{CH}_{3} \mathrm{CH}_{2} \mathrm{OCOH}\right)$ in Orion $\mathrm{KL}$ (Tercero et al. 2013) makes the acetate containing species potential molecules in the interstellar medium. Among them, vinyl acetate $\left(\mathrm{CH}_{3} \mathrm{C}=\mathrm{OOCH}=\mathrm{CH}_{2}\right)$ is a logical molecule to search for since it is related to methyl acetate and it is the simplest unsaturated carboxylic ester after vinyl formate. The line survey of Orion KL from 80 to $280 \mathrm{GHz}$ (Tercero et al. 2010) using the IRAM $30 \mathrm{~m}$ telescope presented initially more than 8000 unidentified lines. Nearly 4000 of them have been identified as lines arising from isotopologues and vibrationally excited states of abundant species (Tercero et al. 2011, 2012; Daly et al. 2013; Demyk et al. 2007; Margulès et al. 2009; Carvajal et al. 2009; López et al. 2014) or other organic molecules (Tercero et al. 2013; Kolesniková et al. 2013, 2014). The study of a cloud such as Orion $\mathrm{KL}$ is a good template that could provide

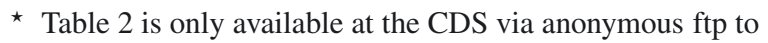
cdsarc.u-strasbg.fr (130.79.128.5) or via http://cdsarc.u-strasbg.fr/viz-bin/qcat?J/A+A/577/A91 important information on the formation of complex organic molecules on the grain surfaces and/or in the gas phase. The ALMA observations are also bringing very sensitive data from the interstellar medium. Therefore, it is important to gather spectroscopic data on potentially detectable organic molecules. The work done by Tercero and collaborators is a first and mandatory step towards interpreting the complex spectra that ALMA will provide for hot cores such as Orion. Taking into account the large number of lines to be identified in Orion, the collaboration between astronomers and spectroscopists is the only way to obtain the maximum scientific output from the line surveys that will be obtained with ALMA.

It was only recently that the rotational spectrum and effective molecular structure of vinyl acetate was first studied. Velino et al. (2009) measured its spectrum between 61 and $77 \mathrm{GHz}$ using the free-jet millimeter absorption technique. The quantum chemical calculations performed by those authors showed that the so-called cis-trans (sp,ap in IUPAC notation) conformer, presented in Fig. 1, is the most stable one. Recently, another work on vinyl acetate in the microwave range from 6 to $40 \mathrm{GHz}$ was performed (Nguyen et al. 2014).

Although the above results provided valuable information about the low-frequency rotational spectrum of vinyl acetate, they cannot be used to accurately predict its frequencies in 


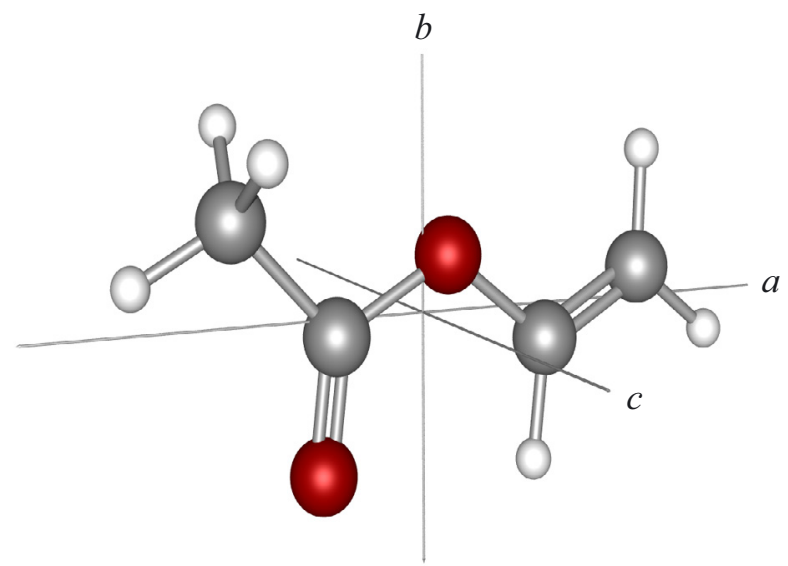

Fig. 1. The lowest energy conformer of vinyl acetate (sp, ap) in the principal axis system.

the millimeter wave domain. There is always some degree of uncertainty involved in predictions of the positions of higher frequency transitions, especially for molecules exhibiting a large amplitude motion with a rather small torsional barrier like vinyl acetate. Possible interstellar detection of vinyl acetate in the millimeter line survey of Orion or another interstellar source should thus be based on transitions measured directly in the laboratory or transitions predicted from a data set that includes higher frequency transitions. Therefore, the objective of the present work is to gather an atlas of line positions and intensities for vinyl acetate up to $305 \mathrm{GHz}$ extending the range of $J$ and $K_{\mathrm{a}}$ to 75 and 16 , respectively, and providing the precise set of the spectroscopic constants that could be used to search for this species in the interstellar medium.

\section{Experiments}

Vinyl acetate (b.p.: $72^{\circ} \mathrm{C}$ ) was purchased from Sigma-Aldrich and used without any further purification. To record the rotational spectrum in the $6-18 \mathrm{GHz}$ frequency range, the sample was loaded into the reservoir of two pulsed nozzles and probed by broadband CP-FTMW spectroscopy. Details about the CP-FTMW spectrometer can be found elsewhere (Mata et al. 2012). Neon carrier gas at a pressure of 2 bar was used for the pulsed jet expansion. To obtain the rotational spectrum of vinyl acetate, 230000 individual free induction decays (four FIDs on each valve cycle) were acquired. A fast Fourier transform with a Kaiser-Bessel window was used to convert the averaged time domain spectrum to the broadband frequency domain spectrum shown in Fig. 2a.

Rotational spectra in the millimeter wave region (125-305 GHz) were recorded by a recently constructed millimeter wave spectrometer at the University of Valladolid. All details concerning the experimental setup (synthesizer, cascade frequency multipliers, detectors) can be found in Daly et al. (2014). Room temperature measurements were carried out at a pressure of 20-30 $\mu$ bar. All spectra were recorded in $1 \mathrm{GHz}$ sections using the frequency modulation technique with $2 f$ lock-in detection, where $f$ is the modulation frequency of $10.2 \mathrm{kHz}$, and modulation depth between $30-50 \mathrm{kHz}$. Frequency accuracy is estimated to be better than $50 \mathrm{kHz}$.

\section{Spectroscopic analysis}

The rotational spectrum of vinyl acetate has two characteristic features. First, the calculated non-zero dipole moment components of $\mu_{a}=-0.05 \mathrm{D}, \mu_{b}=-1.62 \mathrm{D}$, and $\mu_{c}=0.06 \mathrm{D}$ from Velino et al. (2009) points out that only $b$-type transitions are relevant. We should note that the ab initio value of the out-of-plane component of the dipole moment $\mu_{c}$ is likely to be an calculation artefact. Second, vinyl acetate, like other complex organic molecules, exhibits a large amplitude motion, i.e., the torsion of the methyl group relative to the rest of the molecule. The threefold barrier hindering this motion is rather small $\left(V_{3}=152.117(39) \mathrm{cm}^{-1}\right)$ leading to relatively large splittings of the rotational lines into $A$ - and $E$-components even in the ground torsional state $v_{\mathrm{t}}=0$. While only a limited number of $A$ - $E$ doublets of the $b$-type $R$-branch transitions could be measured in the microwave $6-18 \mathrm{GHz}$ region (see Fig. 2a), these transitions dominate in the millimeter wave region. A section of the millimeter wave spectra measured is shown in Fig. 2b, where the $A-E$ doublets for lowest values of the $K_{\mathrm{a}}$ quantum numbers are assigned. The leading $A$ and $E$ lines consist of a pair of $b$-type $R$-branch transitions between degenerate $K_{\mathrm{a}}=0$ and $K_{\mathrm{a}}=1 \mathrm{lev}-$ els, see Fig. 2b. Successive $A-E$ doublets going from the leading one to the left are for increasing $K_{\mathrm{a}}$ and decreasing $J$ transitions and reveal the same type of degeneracies. Figure $2 \mathrm{~b}$ also illustrates the dependence of the $A-E$ splitting magnitude on the $K_{\mathrm{a}}$ quantum number.

Assignment of the low $J$ transitions from 6 to $18 \mathrm{GHz}$ was straightforward, since the supersonic expansion technique allows us to cool down the sample and to observe only the rotational transitions from the lowest energy levels (see Fig. 2a). Transitions measured in the CP-FTMW spectra $(0 \leq J \leq 12$ and $\left.K_{\mathrm{a}} \leq 4\right)$ and those from Velino et al. (2009) $(3 \leq J \leq 25$ and $K_{\mathrm{a}} \leq 5$ ) were weighted with an experimental uncertainty of $5 \mathrm{kHz}$ and $40 \mathrm{kHz}$, respectively, and analyzed together using the internal rotation program BELGI-Cs (Hougen et al. 1994; Kleiner 2010). The principal advantage of the RAM general approach used in the BELGI-Cs code is that takes into account simultaneously $A$ - and $E$-symmetry states and makes it possible to reproduce the experimental data within the experimental accuracy from the microwave to the submillimeter wave range. This first fit enabled us to provide new predictions to facilitate the extension of the assignments of the vinyl acetate rotational transitions into the millimeter wave region. The millimeter wave spectrum of vinyl acetate exhibits higher line density than CP-FTMW spectrum not only because of the higher $J$ and $K_{\mathrm{a}}$ transitions from the $v_{\mathrm{t}}=0$ state, but also from the lowlying excited vibrational states sufficiently populated at the room temperature of the experiment. Loomis-Wood-type plots in the AABS package (Kisiel et al. 2005) were used to guide a search of both $A$ and $E$ lines. An example of this plot is shown in Fig. 3 where all transitions are lined up to the central frequencies of the $A$-component $K_{\mathrm{a}}^{\prime} \leftarrow K_{\mathrm{a}}^{\prime \prime}=1 \leftarrow 0$ transitions and each $K_{\mathrm{a}}^{\prime} \leftarrow K_{\mathrm{a}}^{\prime \prime}$ sequence for both $A$ - and $E$-components then forms a visible stripe running from the lower to the upper values of $J$ quantum numbers.

For consistency, apart from the 62 CP-FTMW and 2220 millimeter wave transitions measured in this work, 72 additional transitions from Velino et al. (2009) and 154 transitions measured by Nguyen et al. (2014) were included in the final fit. Hence, 1378 transitions for the $A$-component and 1130 transitions for the $E$-component were fit with an overall (unitless) standard deviation of 0.905 (root mean square deviation of 5.1 $\mathrm{kHz}$ for the FTMW lines and $42.2 \mathrm{kHz}$ for the millimeter wave lines) using 24 molecular parameters. Table 1 presents all 24 floated torsion-rotation parameters up to the 6th order of RAM model. Among the floated parameters, there are three rotational constants $A^{\mathrm{RAM}}, B^{\mathrm{RAM}}$, and $C^{\mathrm{RAM}}$ as well as the $D_{a b}$ parameter 

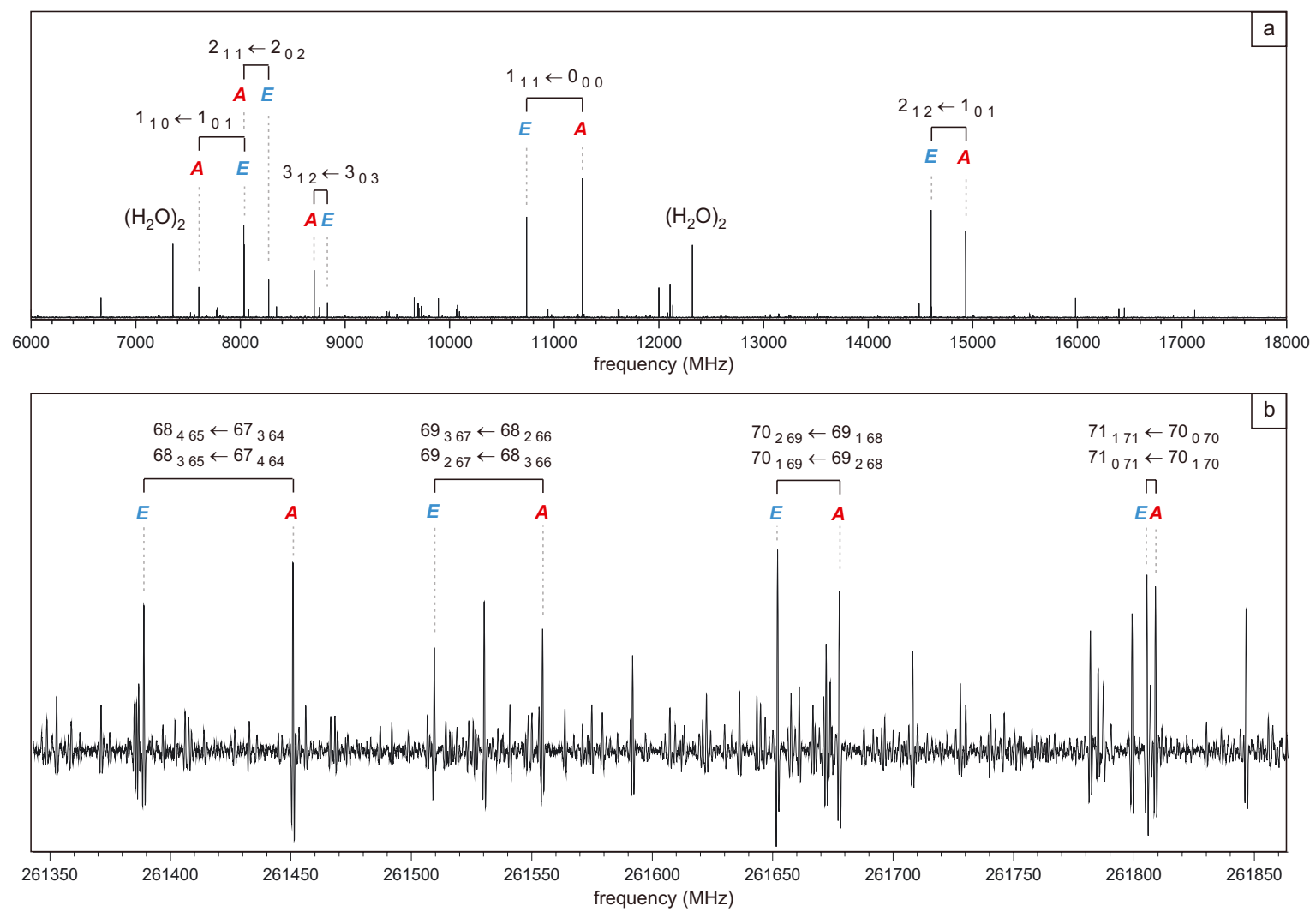

Fig. 2. a) Broadband chirped pulse Fourier transform microwave spectrum (CP-FTMW) from 6 to $18 \mathrm{GHz}$. b) A section of the millimeter wave spectrum of vinyl acetate showing the assignments of the $A$ - and $E$ - components for the ground torsional state $J_{K_{\mathrm{a}}^{\prime} K_{\mathrm{c}}^{\prime}}^{\prime} \leftarrow J_{K_{\mathrm{a}}^{\prime \prime} K_{\mathrm{c}}^{\prime \prime}}^{\prime \prime}$ transitions.

multiplying the $J_{a} J_{b}+J_{b} J_{a}$ operator. The $D_{a b}$ parameter comes from the use of the rho-axis system which is not the principal axis system. $D_{a b}$ is directly related to the angle $\theta_{\mathrm{RAM}}$ between the rho-axis system and the principal axis system (Kleiner 2010). For vinyl acetate, $\theta_{\mathrm{RAM}}=11.83^{\circ}$. Table 1 also provides the values for the centrifugal distortion parameters in the rho-axis system, the barrier height $V_{3}$, the value of the internal rotation constant $F$ and the coupling constant between internal and global rotation $\rho$. Because of the rather low value of the barrier and the extended dataset involving higher $J$ values, it was possible to simultaneously float both $V_{3}$ and $F$. Finally, 11 higher parameters describing the interaction between internal and global rotation ( $D_{a c}, \Delta_{a b}, k_{2}, k_{5}, F_{v}, G_{v}, c_{1}, c_{2}, c_{4}, d_{a b}, D_{a b J}$ ) had to be introduced to achieve the fit reproducing the data within the experimental uncertainty. Line assignments, observed frequencies $v_{\mathrm{obs}}$, calculated frequencies $v_{\text {calc }}$ with the corresponding uncertainties, $v_{\mathrm{obs}}-v_{\text {calc }}$ values, and references of the data sources included in the final fit for the torsional ground state of vinyl acetate are presented in Table 2.

\section{Calculation of intensities}

The line strength calculation is reported here because it is an absolute prerequisite for correct molecular identification in the interstellar medium. For the line strength calculation, the procedure reported for the RAM formalism has already been explained in detail (Hougen et al. 1994; Kleiner 2010). The only values available for the dipole moment components in the principal axis system of vinyl acetate are those obtained from ab initio calculations (see Table 1 in Velino et al. 2009). Under the assumption that the line strengths are only driven by the permanent electric dipole components and have no torsional dependencies (Hougen et al. 1994), the components of the dipole moment in the RAM axis system of $\mu_{a}(\mathrm{RAM})=-0.38 \mathrm{D}$, $\mu_{b}(\mathrm{RAM})=-1.5755 \mathrm{D}$ were used. These values were obtained by rotating the dipole moment under the angle $\theta_{\mathrm{RAM}}=11.83^{\circ}$ between RAM and PAM axis systems. A very small value of the out-of-plane dipole moment component, $\mu_{c}$, was neglected. Calculated line strengths together with lower and upper state energies of vinyl acetate in $v_{\mathrm{t}}=0$ are presented in Table 2 .

\section{Radioastronomical observations}

\subsection{IRAM $30 \mathrm{~m}$ data}

Three species containing the vinyl group have been identified in the interstellar medium: vinyl cyanide (Gardner \& Winnewisser 1975), vinyl alcohol (Turner \& Apponi 2001), and propenal (Hollis et al. 2004). Although the last two have not been detected towards Orion KL, the first, vinyl cyanide, presents a large abundance in this source (López et al. 2014). Therefore, after the detection of methyl acetate in Orion KL (Tercero et al. 2013) and taking into account the large abundance of vinyl species in this source (López et al. 2014), vinyl acetate seems to be a potential candidate for detection in the millimeter line survey with the IRAM 30 m telescope towards Orion KL (Tercero et al. 2010) ranged from 80 to $307 \mathrm{GHz}$ (new data from 280 to $307 \mathrm{GHz}$ have recently been observed). In order to proceed with the search for vinyl acetate, the new spectroscopic data from this work were implemented into the MADEX code (Cernicharo 2012). However, we did not find this species above the confusion limit in those observations. In order to derive an upper limit to its column density, we assumed the same physical conditions as those provided in Tercero et al. (2013) for methyl acetate. 


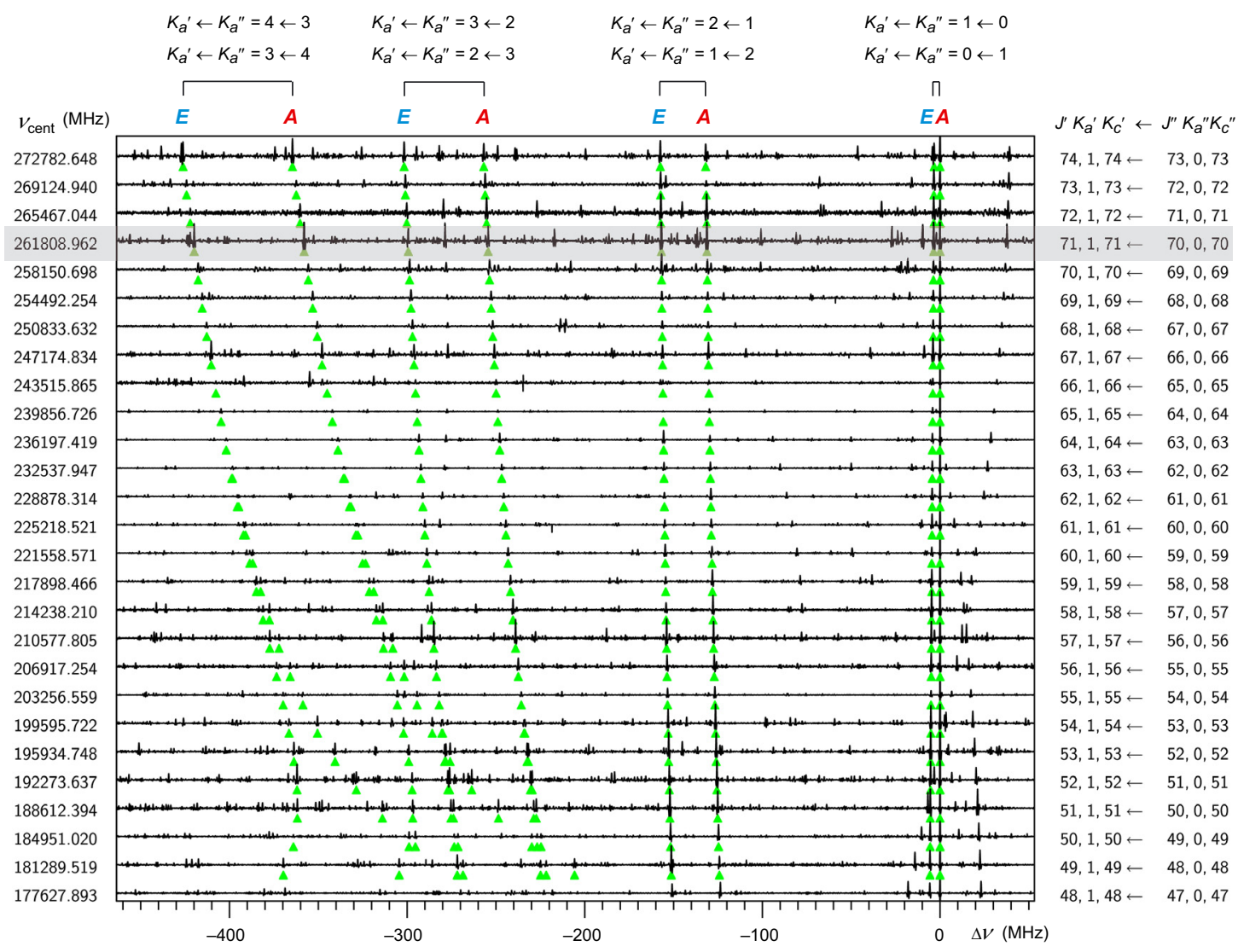

Fig. 3. Loomis-Wood-type plot obtained from the AABS programs package (Kisiel et al. 2005) showing the identification of the stripes corresponding to the $K_{\mathrm{a}}^{\prime} \leftarrow K_{\mathrm{a}}^{\prime \prime}$ sequences of the $b$-type $R$-branch transitions for $K_{\mathrm{a}}^{\prime \prime}=0,1,2,3$, and 4 . Each sequence has two components, $A$ and $E$, due to the methyl internal rotation. Rotational transitions are lined up to the central frequencies $v_{\text {cent }}$ of the $A$-component $K_{\mathrm{a}}^{\prime} \leftarrow K_{\mathrm{a}}^{\prime \prime}=1 \leftarrow 0$ transitions with the rotational quantum numbers indicated on the right side of the diagram. The frequency distance $\Delta v$ from the central frequency is documented on the $x$-axis. Each $A-E$ doublet going to the left from the leading one at $\Delta v=0$ corresponds to increasing $K_{\mathrm{a}}$ and decreasing $J$ transitions. The highlighted horizontal stripe represents the part of the spectrum documented in Fig. 2b. For $J^{\prime \prime}<60$, the degeneracies for the energy levels with $K_{\mathrm{a}}=3$ and $K_{\mathrm{a}}=4$ are lifted and two separate sequences of $K_{\mathrm{a}}^{\prime} \leftarrow K_{\mathrm{a}}^{\prime \prime}=4 \leftarrow 3$ (the left one) and $K_{\mathrm{a}}^{\prime} \leftarrow K_{\mathrm{a}}^{\prime \prime}=3 \leftarrow 4$ (the right one) transitions are recognizable for both $A$ - and $E$-components.

Therefore, we assume that most of the vinyl acetate emission should come from the so-called compact ridge component of this source (see, e.g., Blake et al. 1987, for the different spectral components found in Orion KL with single-dish observations). Using the MADEX code we found a total upper limit to its column density $(A+E$ species $)$ of $(4 \pm 2) \times 10^{14} \mathrm{~cm}^{-2}$ assuming LTE approximation, $v_{\mathrm{LSR}}=8 \mathrm{~km} \mathrm{~s}^{-1}, \Delta v=3 \mathrm{~km} \mathrm{~s}^{-1}$, and the following physical properties for the compact ridge: $T_{K}=150 \mathrm{~K}$, a source diameter of $15^{\prime \prime}$, and a location placed $7^{\prime \prime}$ with respect to the pointing position of the $30 \mathrm{~m}$ data (IRc2). Hence, using the column density value of methyl acetate derived by Tercero et al. (2013), we found an abundance ratio between both species of $N\left(\mathrm{CH}_{3} \mathrm{COOCH}_{3}\right) / N\left(\mathrm{CH}_{3} \mathrm{COOCHCH}_{2}\right) \geq 11$. To compare with other results of the ratio between methyl/vinyl species, the derived value is 1.3 times higher than the total abundance ratio between methyl and vinyl cyanide given in López et al. (2014) in the same source but in another well known component, the so-called hot core. Other interesting ratios that could give us information about the relative abundance of vinyl acetate are those between the methyl/ethyl and ethyl/vinyl abundances of related species. In Orion $\mathrm{KL}$, we found the following values: $N\left(\mathrm{CH}_{3} \mathrm{OH}\right) / N\left(\mathrm{CH}_{3} \mathrm{CH}_{2} \mathrm{OH}\right) \simeq 30$ (Kolesniková et al. 2014); $N\left(\mathrm{CH}_{3} \mathrm{OCOH}\right) / N\left(\mathrm{CH}_{3} \mathrm{CH}_{2} \mathrm{OCOH}\right) \simeq$ 18 (only considering the compact ridge components for the methyl formate column density given in Margulès et al. (2010); the column density of ethyl formate is that given in Tercero et al. 2013); $N\left(\mathrm{CH}_{3} \mathrm{CN}\right) / N\left(\mathrm{CH}_{3} \mathrm{CH}_{2} \mathrm{CN}\right) \simeq 0.55$ (only for the hot core components López et al. 2014), and $N\left(\mathrm{CH}_{3} \mathrm{CH}_{2} \mathrm{CN}\right) / N\left(\mathrm{CH}_{2} \mathrm{CHCN}\right) \simeq 9$ (López et al. 2014). We have to point out that these ratios are derived with data obtained with a single-dish telescope; therefore, a considerable uncertainty arises from the assignation of similar spatial distribution for these species. Moreover, the column density of a single-cloud component could be over/underestimated in the multi-component line profiles observed with the $30 \mathrm{~m}$ telescope. Nevertheless, it seems that the methyl species are more abundant than the ethyl species and, considering that only vinyl cyanide has been detected, the ethyl species could have larger abundances than the vinyl ones. Therefore, it is possible that ethyl acetate could be more abundant than vinyl acetate. Unfortunately, accurate frequencies are not available for ethyl acetate in the millimeter range.

\subsection{ALMA SV data}

In order to further explore the presence of vinyl acetate in Orion KL, we also searched for this species in the ALMA science verification (SV) data ${ }^{1}$ between 213.7 and $246.7 \mathrm{GHz}$.

\footnotetext{
1 http://almascience.eso.org/almadata/sciver/ OrionKLBand6/
} 

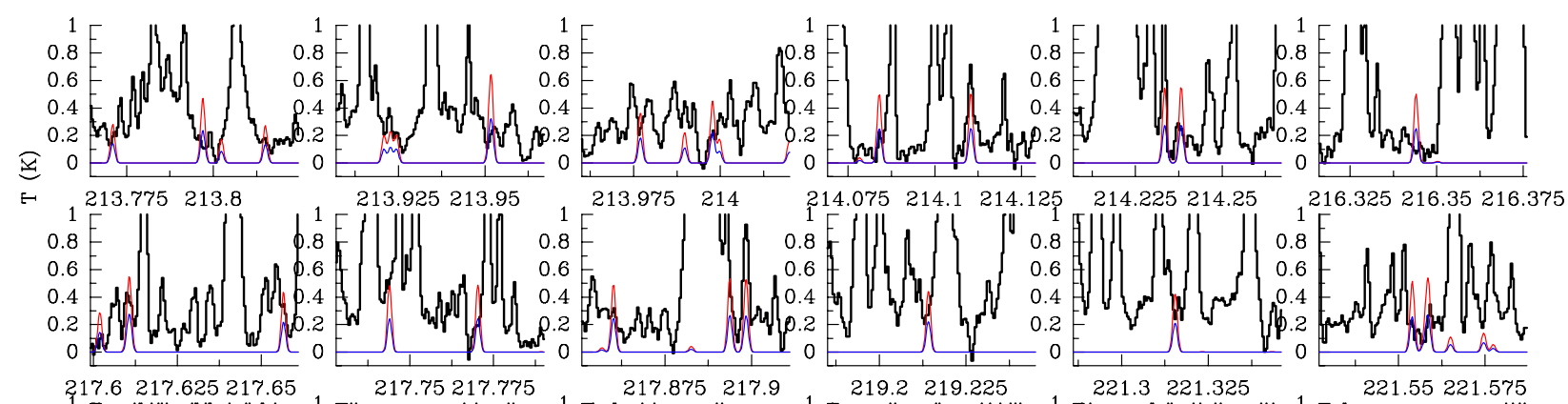

216.325216 .35216 .375
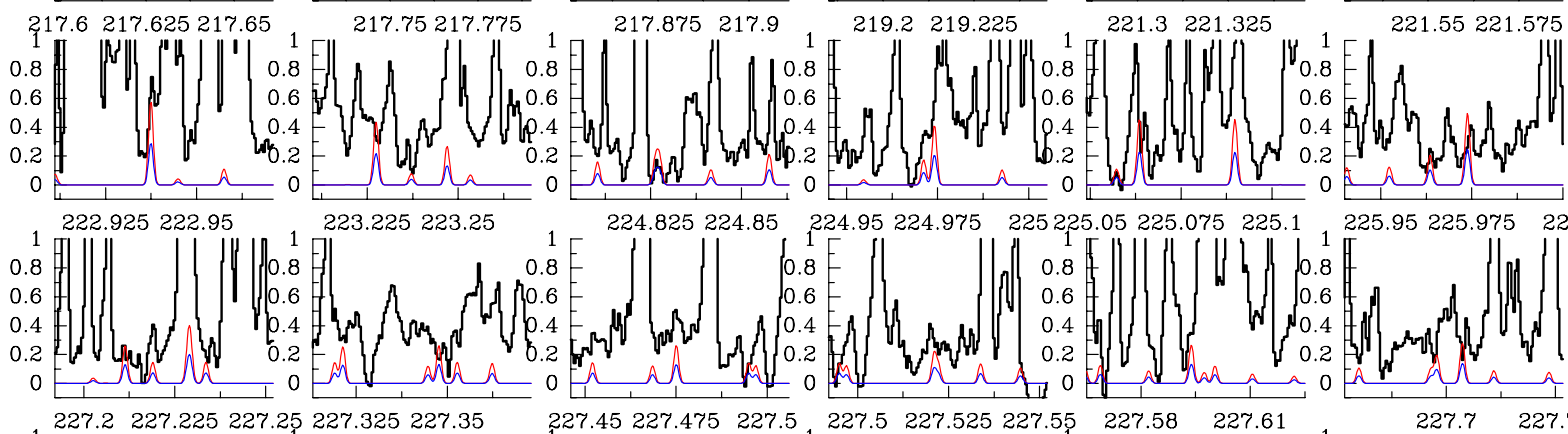

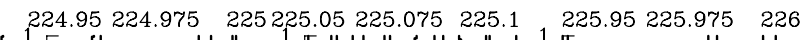

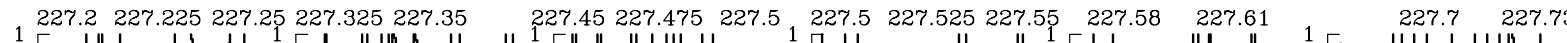
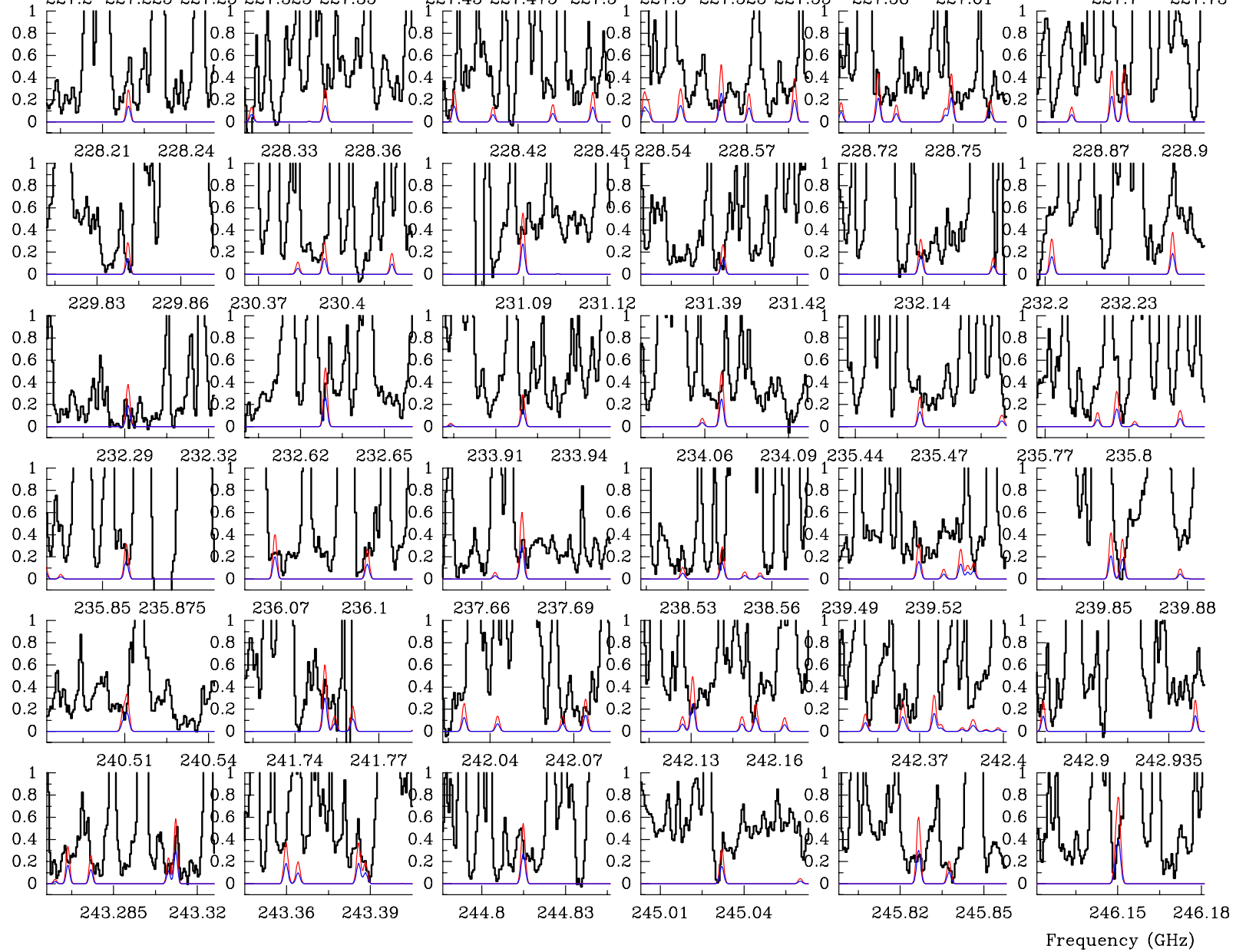

Fig. 4. Lines of vinyl acetate, $\mathrm{CH}_{3} \mathrm{COOCHCH}_{2}$, towards the compact ridge of Orion KL. The black histogram is the averaged spectrum over $5 \times 5$ pixels around the methyl acetate peak (see text) of the ALMA SV data (see text for references); these data have a $0.5 \mathrm{MHz}$ of spectral resolution. The thin red line is the synthetic spectrum assuming a column density of $2 \times 10^{15} \mathrm{~cm}^{-2}$ for vinyl acetate $(A+E$ species). The thin blue line is the model for vinyl acetate with a column density of $1 \times 10^{15} \mathrm{~cm}^{-2}(A+E$ species); this model corresponds to the given column density for the tentative detection of vinyl acetate (see text). A $v_{\text {LSR }}$ of $7 \mathrm{~km} \mathrm{~s}^{-1}$ is assumed.

First, we determined the position where methyl acetate peaks. As expected, it is within the compact ridge component $\left(\alpha_{2000.0}=05^{\mathrm{h}} 35^{\mathrm{m}} 14.117^{\mathrm{s}}, \delta_{2000.0}=-05^{\circ} 22^{\prime} 36.54^{\prime \prime}\right)$. We extracted the ALMA SV averaged spectrum of $5 \times 5$ pixels $\left(0.95^{\prime \prime} \times 0.95^{\prime \prime}\right)$ around this position. In addition to the higher sensitivity, one of the advantages of ALMA with respect to single-dish telescopes (to search for new molecules) is the drastic reduction of the line confusion limit. Whereas in the $30 \mathrm{~m}$ 
Table 1. Molecular constants in the rho-axis system of vinyl acetate obtained from the global fit using program BELGI-Cs.

\begin{tabular}{lllr}
\hline \hline Constant $^{a}$ & Unit & Operator $^{b}$ & Value $^{c}$ \\
\hline$A^{\mathrm{RAM}}$ & $\mathrm{MHz}$ & $J_{a}^{2}$ & $9076.272(41)$ \\
$B^{\mathrm{RAM}}$ & $\mathrm{MHz}$ & $J_{b}^{2}$ & $2544.414(60)$ \\
$C^{\mathrm{RAM}}$ & $\mathrm{MHz}$ & $J_{c}^{2}$ & $1832.239(42)$ \\
$D_{a b}$ & $\mathrm{MHz}$ & $\left\{J_{a}, J_{b}\right\}$ & $-1431.559(96)$ \\
$\Delta_{J}^{\mathrm{RAM}}$ & $\mathrm{kHz}$ & $-J^{4}$ & $0.2742(36)$ \\
$\Delta_{J K}^{\mathrm{RAM}}$ & $\mathrm{kHz}$ & $-J_{a}^{2} J^{2}$ & $-0.195(16)$ \\
$\Delta_{K}^{\mathrm{RAM}}$ & $\mathrm{kHz}$ & $-J_{a}^{4}$ & $11.421(23)$ \\
$\delta_{J}^{\mathrm{RAM}}$ & $\mathrm{kHz}$ & $-2 J^{2}\left(J_{b}^{2}-J_{c}^{2}\right)$ & $0.0834(18)$ \\
$\delta_{K}^{\mathrm{RAM}}$ & $\mathrm{kHz}$ & $-\left\{J_{a}^{2},\left(J_{b}^{2}-J_{c}^{2}\right)\right\}$ & $0.4721(70)$ \\
$\Phi_{J}^{\mathrm{RAM}}$ & $\mathrm{mHz}$ & $J^{6}$ & $0.0390(30)$ \\
$V_{3}$ & $\mathrm{~cm}-1$ & $(1 / 2)(1-\cos 3 \gamma)$ & $152.117(39)$ \\
$F$ & $\mathrm{~cm}-1$ & $J_{\gamma}^{2}$ & $5.3881(10)$ \\
$\rho$ & $\mathrm{unitless}$ & $J_{a} J_{\gamma}$ & $0.0460572(17)$ \\
$D_{a c}$ & $\mathrm{MHz}$ & $\left\{J_{a}, J_{c}\right\}(\sin 3 \gamma)$ & $-24.60(58)$ \\
$\Delta_{a b}$ & $\mathrm{MHz}$ & $J_{\gamma}^{2}\left(J_{a} J_{b}+J_{b} J_{a}\right)$ & $-0.520(11)$ \\
$k_{2}$ & $\mathrm{MHz}$ & $J_{\gamma}^{2} J_{a}^{2}$ & $0.3947(87)$ \\
$k_{5}$ & $\mathrm{MHz}$ & $(1-\cos 3 \gamma) J_{a}^{2}$ & $81.29(12)$ \\
$F_{v}$ & $\mathrm{MHz}$ & $(1-\cos 3 \gamma) J^{2}$ & $-3.505(48)$ \\
$G_{v}$ & $\mathrm{MHz}$ & $J_{\gamma}^{2} J^{2}$ & $0.0980(29)$ \\
$c_{1}$ & $\mathrm{MHz}$ & $2 J_{\gamma}^{2}\left(J_{b}^{2}-J_{c}^{2}\right)$ & $0.0119(26)$ \\
$c_{2}$ & $\mathrm{MHz}$ & $(1-\cos 3 \gamma)\left(J_{b}^{2}-J_{c}^{2}\right)$ & $-2.894(99)$ \\
$c_{4}$ & $\mathrm{kHz}$ & $J_{\gamma}\left\{J_{a},\left(J_{b}^{2}-J_{c}^{2}\right)\right\}$ & $5.08(22)$ \\
$d_{a b}$ & $\mathrm{MHz}$ & $(1-\cos 3 \gamma)\left\{J_{a}, J_{b}\right\}$ & $-13.51(19)$ \\
$D_{a b J}$ & $\mathrm{kHz}$ & $\left\{J_{a}, J_{b}\right\} J^{2}$ & $0.559(16)$ \\
\hline & & &
\end{tabular}

Notes. ${ }^{(a)}$ All constants refer to the rho-axis system; therefore, the inertia tensor is not diagonal and the constants cannot be directly compared to those of the principal axis system. ${ }^{(b)} J_{a}, J_{b}, J_{c}$ are the components of the overall rotation angular momentum, $J_{\gamma}$ is the angular momentum of the internal rotor rotating around the internal rotor axis by an angle $\gamma$, and $\{u, v\}$ is the anti commutator $u v+v u$. The product of the constant and operator from a given row yields the term actually used in the vibrationrotation-torsion Hamiltonian, except for $F, \rho$, and $A$, which occur in the Hamiltonian in the form $F\left(J_{\gamma}-\rho J_{a}\right)^{2}+A J_{a}^{2}$. ${ }^{(c)}$ Values of the parameters from the present fit. Statistical uncertainties are shown as one standard uncertainty in the last digits.

data we obtained the averaged spectrum over a beam diameter ranged from $30^{\prime \prime}$ to $9^{\prime \prime}$, the synthetic beam of the ALMA SV data is $1.90^{\prime \prime} \times 1.40^{\prime \prime}$. Therefore, in the $30 \mathrm{~m}$ data, the spectrum is a mix of all molecules from all source components giving rise to a high level of blending of the lines. Taking the mentioned advantage, we could detect tentatively vinyl acetate towards the compact ridge position of Orion KL with the ALMA SV data (see Fig. 4). In Fig. 4, the blue model provides the physical and chemical parameters that fit the observational ALMA data. The assumed parameters for this model are a source size of 3" (better adapted to the ALMA synthetic beam), $v_{\text {LSR }}=7 \mathrm{~km} \mathrm{~s}^{-1}, \Delta v=2 \mathrm{~km} \mathrm{~s}^{-1}$, and $T_{K}=150 \mathrm{~K}$. Using the MADEX code in LTE approximation, we obtained a column density $(A+E$ species $)$ of $1 \times 10^{15} \mathrm{~cm}^{-2}$. The red model uses the same parameters but a column density of $2 \times 10^{15} \mathrm{~cm}^{-2}$, above which we could start to say that lines were missing or mismatched. We consider that the blue model is better adapted to the data, so we only provide a tentative detection. Although many lines are below the detection limit for the blue model, we found that several lines are above this limit and no missing lines are found. To verify this tentative detection, we performed a synthetic spectrum with the same physical conditions but with the IRAM $30 \mathrm{~m}$ observations in order to search again for this molecule in these data. The new modeled line intensities appear below the detection limit, because the beam dilution factor for the $30 \mathrm{~m}$ is much higher than the gain by the higher column density obtained by ALMA. Therefore, no missing lines were found in the single-dish data confirming the tentative detection provided above.

Owing to the physical conditions of Orion KL, the maximum line density appears at the $1 \mathrm{~mm}$ window, i.e., at the wavelengths of the available observations with ALMA. Therefore, the confusion limit, even for filtered observations of the compact ridge is enough to hide the weak lines of vinyl acetate. Observations with ALMA at $2 \mathrm{~mm}$ could give us the major opportunity to confirm the detection of vinyl acetate in Orion KL.

Acknowledgements. The research leading to these results has received funding from the European Research Council under the European Union's Seventh Framework Programme (FP/2007-2013)/ERC-2013-SyG, Grant Agreement No. 610256 NANOCOSMOS, Ministerio de Ciencia e Innovación (Grants CTQ2010-19008, CTQ2013-40717-P, and Consolider-Ingenio 2010 CSD200900038 program "ASTROMOL") and Junta de Castilla y León (Grants VA070A08 and VA175U13). I.K. would like to thank the French PCMI (Programme National de Physique Chimie du Milieu Interstellaire).

\section{References}

Belloche, A., Garrod, R. T., Müller, H. S. P., et al. 2009, A\&A, 499, 215

Belloche, A., Müller, H. S. P., Menten, K. M., Schilke, P., \& Comito, C. 2013, A\&A, 559, A47

Blake, G. A., Sutton, E. C., Masson, C. R., \& Philips, T. H. 1987, ApJ, 315, 621 Carvajal, M., Margulès, L., Tercero, B., et al. 2009, A\&A, 500, 1109

Cernicharo, J. 2012, in ECLA-2011: Proc. European Conference on Laboratory Astrophysics, Laboratory Astrophysics and Astrochemistry in the Herschel/ALMA Era, 58, 251

Daly, A. M., Bermúdez, C., López, A., et al. 2013, ApJ, 768, 81

Daly, A., Kolesniková, L., Mata, S., \& Alonso, J. 2014, J. Mol. Spectr., 306, 11

Demyk, K., Mader, H., Tercero, B., et al. 2007, A\&A, 466, 255

Gardner, F. F., \& Winnewisser, G. 1975, ApJ, 195, L127

Hollis, J. M., Jewell, P. R., Lovas, F. J., Remijan, A., \& Møllendal, H. 2004, ApJ, 610, L21

Hougen, J., Kleiner, I., \& Godefroid, M. 1994, J. Mol. Spectr., 163, 559

Kisiel, Z., Pszczółkowski, L., Medvedev, I. R., et al. 2005, J. Mol. Spectr., 233, 231

Kleiner, I. 2010, J. Mol. Spectr., 260, 1

Kolesniková, L., Daly, A., Alonso, J., Tercero, B., \& Cernicharo, J. 2013, J. Mol. Spectr., 289, 13

Kolesniková, L., Tercero, B., Cernicharo, J., et al. 2014, ApJ, 784, L7

López, A., Tercero, B., Kisiel, Z., et al. 2014, A\&A, 572, A44

Margulès, L., Motiyenko, R., Demyk, K., et al. 2009, A\&A, 493, 565

Margulès, L., Huet, T. R., Demaison, J., et al. 2010, ApJ, 714, 1120

Mata, S., Pena, I., Cabezas, C., López, J., \& Alonso, J. 2012, J. Mol. Spectr., 280, 91

Nguyen, H. V. L., Jabri, A., Van, V., \& Stahl, W. 2014, J. Phys. Chem. A, 118, 12130

Tercero, B., Cernicharo, J., Pardo, J. R., \& Goicoechea, J. R. 2010, A\&A, 517, A96

Tercero, B., Vincent, L., Cernicharo, J., Viti, S., \& Marcelino, N. 2011, A\&A, 528, A26

Tercero, B., Margulès, L., Carvajal, M., et al. 2012, A\&A, 538, A119

Tercero, B., Kleiner, I., Cernicharo, J., et al. 2013, ApJ, 770, L13

Turner, B. E., \& Apponi, A. J. 2001, ApJ, 561, L207

Velino, B., Maris, A., Melandri, S., \& Caminati, W. 2009, J. Mol. Spectr., 256, 228 